\title{
SAR Image Categorization Using Parametric and Nonparametric Approaches Within a Dual Tree CWT
}

\author{
Peter Planinšič, Member, IEEE, Jagmal Singh, and Dušan Gleich, Member, IEEE
}

\begin{abstract}
This letter presents synthetic aperture radar (SAR) image classification based on feature descriptors within the discrete wavelet transform (DWT) domain using parametric and nonparametric features. The DWT enables an efficient multiresolution description of SAR images due to its geometric and stochastic features. A 2-D DWT, a real 2-D oriented dual tree wavelet transform (2-D RODTWT) and an oriented dual tree complex wavelet transform (2-D ODTCWT) were used for the estimation of subband features. First and second moments, entropy, coding gain, and fractal dimension were used for the nonparametric approach. A parametric approach considers a Gauss Markov Random Field model for feature extraction. A database with 2000 images representing 20 different classes with 100 images per class was used for classification efficiency assessment. Several SAR scenes were divided into small patches with dimension of $200 \times 200$ pixels. $10 \%$ and $20 \%$ of the test images per class were used during the learning stage. Supervised learning using a support vector machine was used for all experiments. The experimental results showed that the proposed methods had superior performances compared with (GLCM) and log comulants of Fourier transform. Amongst the proposed methods, the nonparametric features within oriented dual tree complex wavelet transform gave the best results for classes when categorizing SAR images.
\end{abstract}

Index Terms-Data mining, feature extraction, image texture analysis, support vector machines (SVMs), wavelet transforms.

\section{INTRODUCTION}

$\mathbf{T}$ HERE are two main approaches for scene description and classification called parametric and nonparametric approaches. Parametric approaches use theoretical scene models [1], [2], the parameters of which are estimated from the available data under given model assumption. Nonparametric approaches use objective parameters which are estimated from original or transformed data [3], [4]. In [3] feature descriptors (first- and second-order statistical moments, Spectral Centroid, Spectral Flux, Spectral Rolloff, Cepstral Coefficients) for synthetic aperture radar (SAR) image patches were computed within the Fourier domain. The nonparametric feature based classification methods are also well-known within optical and remote sensing communities [5] and content-based image retrieval in multimedia [6]. The feature descriptor, based on log

Manuscript received October 14, 2013; revised January 20, 2014; accepted February 13, 2014.

P. Planinšič and D. Gleich are with the University of Maribor, Faculty of Electrical Engineering and Computer Science, Laboratory for Signal Processing and Remote Control, Maribor 2000, Slovenia.

J. Singh is with the DLR, Earth Observation Center, 82234 Wessling, Germany.

Digital Object Identifier 10.1109/LGRS.2014.2308328 comulants of Fourier coefficients [4] were also applied to the same database and those results have been used in this letter for comparison purposes. The dictionary learning approaches to the image filtering was proposed within a compressed sensing community [7]-[9].

This letter proposes the use of parametric and nonparametric feature descriptors within different types of discrete wavelet transformations for patch-scene classification. The features for categorization obtained in this letter were compared with those features obtained with the Gray Level Co-occurrence Matrix (GLCM) of SAR-patches [10], the feature descriptors in the Fourier domain [3] and the log comulants of Fourier coefficients [4]. The Support Vector Machine (SVM) classifier [11] was used for supervised learning in all experiments.

\section{Dual Tree Complex Wavelet Transform}

Wavelets constructed using complex filter banks during image processing were originally proposed in [12] within the framework of the Daubechies orthogonal filters banks and later generalized to complex wavelet transform (CWT) [13]. CWT is a complex-valued extension to the standard real-valued discrete wavelet transform (DWT). CWT has an analytical wavelet function, by which the imaginary part is the Hilbert transform of the real part. By the implementation of CWT with finite impulse response filters, this condition is fulfilled approximately. In contradiction to classical DWT it does not suffer from aliasing and oscillations, provides a high degree of shift-invariance magnitude and orientation in two and higher dimensions, which are the properties desired for pattern and texture classification and recognition. It exhibits redundancy $2^{d}$ (where $d$ is the dimension of the signal being transformed), which is significantly lower than undecimated DWT.

This letter uses the dual-tree approach for complex wavelet transform (DTCWT), which calculates the complex transform of a signal using two separate real DWT-decompositions (tree a and tree $\mathrm{b}$ ). If the filters used in tree $a$ are specifically designed differently from those in tree $b$ then it is possible for one DWT to produce the real coefficients and the other the imaginary. More exactly, to really achieve the desired analytical properties, the filters in the trees are interchanged at each decomposition level and during the first stage any perfect reconstruction filters can be used within each tree. In two dimensions, 6 orientations are obtained by using 2-D real oriented dual tree wavelet transform (2-D RODTWT) and 12 orientations by using 2-D ODTCWT. The details regarding the implementing a DTCWT can be found in [13]. 


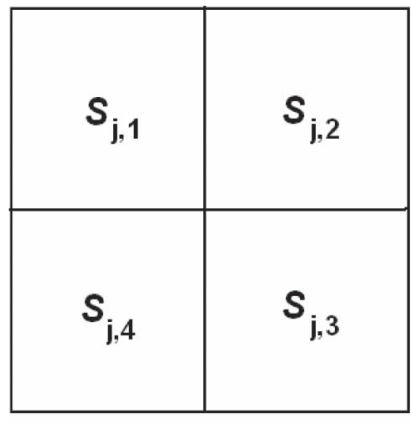

(a)

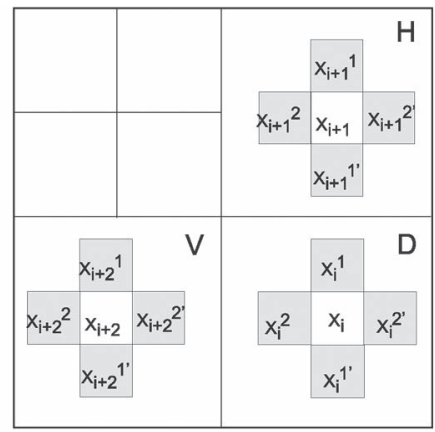

(b)
Fig. 1. (a) Subbands $S_{j, i}$ obtained at each level using separable 2D-DWT. (b) Wavelet coefficients forming a first-order GMRF.

\section{FeAture Descriptors Within Discrete WAVELET TRANSFORM}

Scene classes from SAR image patches can be determined using different descriptors (feature vectors). Descriptors within wavelet domain are very appropriate and a natural choice due to the multi-resolution properties of wavelet transform and on the other hand deterministic or stochastic self-similarity properties of many natural and urban amplitude SAR-image's scenes. The image is deterministic, if it is invariant to dilatations and compression and it is stochastic self-similar, if it is statistically invariant for dilatations and compression [14]. Different wavelet transforms can be used from continuous (CWT) to discrete wavelet transform (DWT) [15] and discrete wavelet packets transform (DWPT) [16]. DWPT can be considered as the generalization of DWT, where also the detail wavelet coefficients are further decomposed with low-pass and high-pass filters. It was found in [17] that the DWPT tree for amplitude SARimages reduces to a DWT (Laplacian pyramid) using Shannon's Entropy criteria for any decision on further decomposition. For this reason and for the computational efficiency we decided to use feature descriptors within discrete wavelet transform domain. As it will be shown later, TerraSAR-X images with their high resolution properties enable classification of scenes which on bigger regions and globally look similar or almost equal. The classification efficiency is improved by using feature vectors with descriptors within DWT-domain.

\section{A. Nonparametric Feature Extraction}

The nonparametric feature extraction is performed using an image patch of $M \times N=200 \times 200$ pixels. Three scenarios were considered using: DWT, real oriented dual tree WT (RODTWT) and oriented dual tree complex wavelet transform (ODTCWT). Descriptors were evaluated for each subband of the wavelet transform with $j$ decomposition levels. The patch with dimension $M \times N$ pixels is characterized by the feature vector, with descriptors for all subbands $S_{j, i}$. Subband $S_{j, 1}$ is obtained by low-pass filtering in horizontal and vertical directions (LL), subband $S_{j, 2}$ by low-pass filtering in horizontal and high-pass filtering in the vertical direction (LH), subband $S_{j, 3}$ by high-pass filtering in both direction ( $\mathrm{HH}$ ), and subband $S_{j, 4}$ by high-pass filtering in horizontal and low-pass filtering in the vertical direction (HL). Subbands are depicted in Fig. 1(a).

A feature vector within wavelet transform consists of subbands variances $\delta_{j, i}^{2}$, mean values $\mu_{j, i}, L 2$-norm $\left\|S_{j, i}\right\|_{2}$ and $L 1$-norm $\left\|S_{j, i}\right\|_{1}$, entropy, coding gain and fractal dimension.
Thus, the variance of subbband $S_{j, i}$ with dimension $N_{j} \times$ $M_{j}$ is given by

$$
\delta_{j, i}^{2}=\frac{1}{M_{j} \cdot N_{j}} \sum_{m=0}^{M_{j}-1} \sum_{n=0}^{N_{j}-1}\left(S_{j, i}[m, n]-\mu_{j, i}\right)^{2}
$$

where $S_{j, i}[m, n]$ represents a wavelet coefficient with indexes $m, n$ and $\mu_{j}$ is the subband mean given by

$$
\mu_{j, i}=\frac{1}{M_{j} \cdot N_{j}} \sum_{m=0}^{M_{j}-1} \sum_{n=0}^{N_{j}-1} S_{j, i}[m, n] .
$$

The second-order norm is given by

$$
\left\|S_{j, i}\right\|_{2}=\frac{1}{M_{j} \cdot N_{j}} \sum_{m=0}^{M_{j}-1} \sum_{n=0}^{N_{j}-1}\left|S_{j, i}[m, n]\right|^{2}
$$

and the first-order norm is given by

$$
\left\|S_{j, i}\right\|_{1}=\frac{1}{M_{j} \cdot N_{j}} \sum_{m=0}^{M_{j}-1} \sum_{n=0}^{N_{j}-1}\left|S_{j, i}[m, n]\right|^{1} .
$$

From the information point of view, the entropy of subband is the measure of information contained within the subband. There are many types of entropy, however we decided on the most common Shannon's' entropy given by

$$
E_{j, i}=\sum_{k} p_{k} \log _{2}\left(1 / p_{k}\right)
$$

where $p_{k}$ is the probability of the pixels with value $S_{k}$ occurring within subband $S_{j, i}$.

Another important descriptor is patch smoothness; it can be estimated using a wavelet spectrum (variances versus scale) flatness, which can be further estimated using coding gain. A coding gain of the patch can be calculated from the variances of subbands within the DWT-domain using the expression:

$$
c g=\frac{\prod_{j=1}^{J} \prod_{i=1}^{3} \alpha_{j . i} \cdot \sigma_{j, i}^{2}}{\prod_{j=1}^{J} \prod_{i=1}^{3}\left(\sigma_{j, i}^{2}\right)^{\alpha_{j, i}}}
$$

where $a_{j, i}<1$ is the relative area of the subband with subscripts $j, i$. Note, that the sum of all relative areas is equal to one. A Rate-Distortion (R-D) based categorization can be obtained by using coding gain $c_{g}$ or directly by measuring the R-D-slope using DWT-based lossy compression.

Fractal dimension is also a very important descriptor of self similarity for a patch scene with stochastic or deterministic geometrical self-similar structures. Wavelet-based analysis was shown to be very natural for mathematical description and calculation of fractal dimensions and was therefore included in our research. In this letter, the fractal dimension was estimated using variances of detail subbands. The variance $\delta_{j}^{3}$ of detailed subband $S_{j, 3}$ namely obey a power law of the scale $j$ and can therefore be used to calculate the Hurst parameter $H$ [18] given by

$$
\sigma_{j, 3}^{2}=\frac{K}{\left(2^{j}\right)^{2 H+2}}=\frac{K}{\left(2^{j}\right)^{\beta}}
$$

where the constant $K$ depends on the type of used wavelet function $\psi_{j}^{3}(t)$. H can be calculated from the slope of the linear 
curve obtained by plotting the logarithm of detailed subband variances versus scale $j$, given by

$$
\log _{2} \sigma_{j, 3}^{2}=\beta \cdot j+K \text {. }
$$

The fractal dimension $D$ is related to the Hurst parameter $H$ by the expression [18]

$$
D=E d+1-H
$$

where $E d$ is the Euclid geometrical dimension, for images being equal to 2 .

\section{B. Parametric Feature Extraction}

The proposed method models an image using a Gauss-Markov Random Field (GMRF) model, which can be defined as a generalized Gauss-Markov random field within wavelet transform [20]:

$$
\begin{aligned}
& p\left(x_{i} \mid \theta\right)=\frac{\nu \eta\left(\nu, \sigma_{x}\right)}{2 \Gamma(1 / \nu)} \\
& \times \exp \left(-\left[\eta\left(\nu, \sigma_{x}\right)\left|x_{i}-\sum_{j \in \zeta} \theta_{j}\left(x_{i}^{j}+x_{i}^{j \prime}\right)\right|\right]^{\nu}\right)
\end{aligned}
$$

where $\nu$ is the shape factor, $\sigma_{x}$ is a standard deviation of approximated image model, $\Gamma$ (.) represents Gamma function, $x_{i}$ represents the observed wavelet coefficient and $\eta($.$) is given by$

$$
\eta\left(\nu, \sigma_{x}\right)=\sigma_{x}^{-1} \sqrt{\frac{\Gamma(3 / \nu)}{\Gamma(1 / \nu)}} .
$$

The parameter $\theta$ represents the texture parameter of the GMRF and is usually iteratively estimated using the secondorder Bayesian inference. The GMRF characterizes the spatial statistical dependencies of 2-D data by a symmetric set called the neighbor set or neighborhood system. The sites within the 2-D lattice $\Omega$ are related to one another via a neighborhood system. The neighborhood system in $\Omega$ is defined as $N=$ $\left\{N_{s} \mid \forall s \in \Omega\right\}$, where $N_{s}$ is the set of sites neighboring $s$. The neighboring set for a first-order MRF is defined as $N=\{(0,-1),(0,1),(-1,0),(1,0)\}$ and for a secondorder $\quad N=\{(0,-1),(0,1),(-1,0),(1,0),(-1,1),(1,-1)$, $(-1,-1),(1,1)\}$ and $N_{s}=\{s+r, r \in N\}$. The clique of the neighbor set $N_{s}$ is defined as a subset of sites in $\Omega$. It consists of either a single site or a pair of neighboring sites, and so on. The type of clique is determined by its size, shape, and orientation. We consider the second and higher orders using two-pixel cliques consisting of two facing neighbors' $x_{s+r}$ and $x_{s-r}$.

The image model is characterized using a variance and texture parameter $\theta$, which represents a generalized GMRF model within a neighborhood $\zeta$. The formation of neighboring pixels is shown in Fig. 1(b). Inter-subband and intra-subband relations were used to define six cliques in the following way. Two cliques are formed around the observed wavelet coefficient at a subband level, and four cliques are considered from the corresponding subbands at the same decomposition level, as shown in Fig. 1(b). The unknown parameters $\theta$ and $\sigma_{x}^{2}$ can be estimated using the least square estimate [21], [22], as given here

$$
\begin{aligned}
\theta & =\left(\mathbf{G}^{T} \mathbf{G}\right)^{-1} \mathbf{G}^{T} \mathbf{X} \\
\sigma_{x}^{2} & =\frac{1}{G_{N}-G_{M}}\left(\mathbf{X}^{T} \mathbf{X}-\mathbf{X}^{T} \mathbf{G}\left(\mathbf{G}^{T} \mathbf{G}\right)^{-1} \mathbf{G}^{T} \mathbf{X}\right)
\end{aligned}
$$

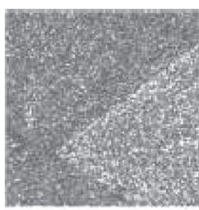

C01-Grassland

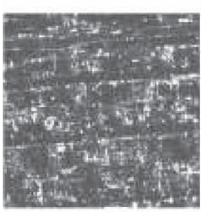

C05-Urban-01

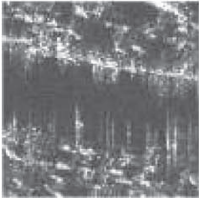

C09-Urban water-channel

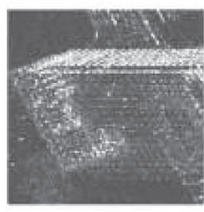

C13Skyscrapers

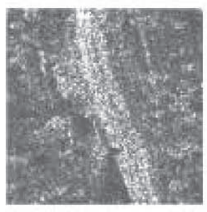

C17-Train tracks

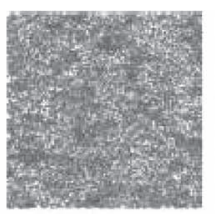

C02-Forest

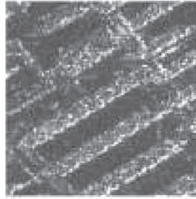

C06-Urban-02

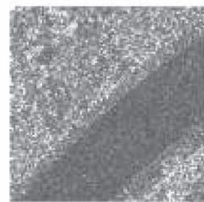

C10-Natural water-channel

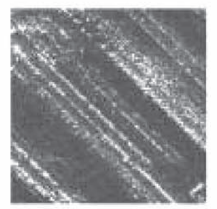

C14-Industrial complex

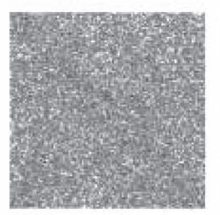

C18-Sandy plains

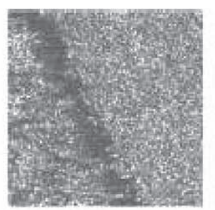

C03-Mixed vegetation

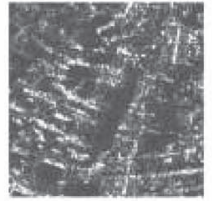

C07-Urban-03

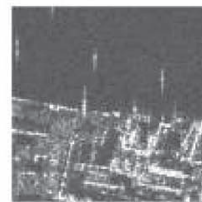

C11-Urban coastline

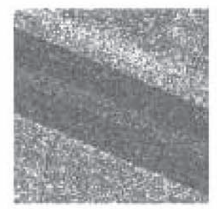

C15-Airstrip

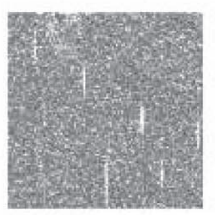

C19-Buoy in Sea

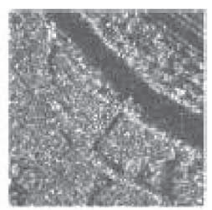

C04-Veg. and a water-channel

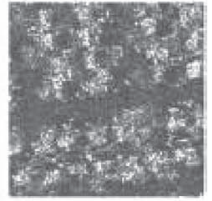

C08-Urban-04

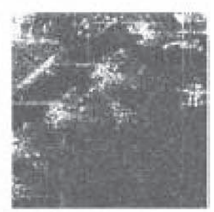

C12-Aircraft stands

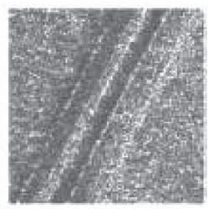

C16-Highway

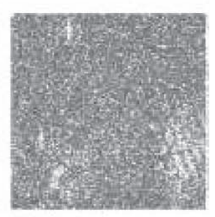

C20-

Ambiguities
Fig. 2. Examples of the SAR image patches (of size $200 \times 200$ pixels) in different object/texture categories. The image patches have been obtained from SLC VHR Spotlight TerraSAR-X images from different areas in order to include the diversity of objects/textures when analyzing feature descriptors.

where $\mathbf{X}$ represents the observed coefficients within a window of $3 \times 3$ pixels and $\mathbf{G}$ consists of neighboring coefficients attributed to each coefficient $x_{i}$ within a window of $3 \times 3$ pixels. The matrix $\mathbf{G}$ has a size of $G_{N} \times G_{M}$ elements. An element $G_{i}$ of a vector $\mathbf{G}$ can be written as

$$
\begin{aligned}
G_{i}^{T}=\left[x_{i}^{1}+x_{i}^{1^{\prime}},\right. & x_{i}^{2}+x_{i}^{2^{\prime}}, x_{i+1}^{1}+x_{i+1}^{1^{\prime}}, x_{i+1}^{2} \\
& \left.+x_{i+1}^{2^{\prime}}, x_{i+2}^{1}+x_{i+2}^{1^{\prime}}, x_{i+2}^{2}+x_{i+2}^{2^{\prime}}\right] .
\end{aligned}
$$

The value $x$ represents an observed wavelet coefficient.

\section{EXPERIMENTAL RESUlts}

\section{A. Database}

A database of 2000 patches was generated within an image size of $200 \times 200$ pixels using SLC VHR Spotlight TerraSAR-X images. Each patch covered approximately $200 \mathrm{~m}^{2}$ of ground. This size can be generally used to define a particular category, as it is large enough to contain the contextual information 
needed to define an object's structure in the case of HR yet also suitable for texture computation of homogeneous areas. The presented experimental database was compriseed 20 welldefined object/texture categories with 100 patches in each category. Care was taken to generate a database with diverse textures. Examples of one patch from each category are shown in Fig. 2. These categories include land-cover topologies (C01Grassland, C02- Forest, C03-Mixed vegetation, C04-Vegetation and a water channel, and C18-Sandy plains), urban areas with a particular texture (C05-Urban-01, C06-Urban-02, C07-Urban03, and C08-Urban-04), urban and natural water channels (C09Urban water-channel and C10-Natural water-channel), regions with strong scatterers along with the surfaces exhibiting specular reflection (C11-Urban coastline and C12-Aircraft stands), regions with very strong scatterers with multiple bounces (C13Skyscrapers and C14-Industrial complex), and other special categories like C15-Airstrip, C16-Highway, and C17-Train tracks. Two categories from the sea-water region (C19-Buoy in Sea and C20-Ambiguities) were also considered. This database was generated manually, thus, it could be used as a ground truth for the assessment of various algorithms.

\section{B. Assessment}

The second-order statistics of the amplitude SAR image patches were computed using GLCMs, extracting 12 textural features and used for a comparative assessment. This implementation allowed four options for the orientation (0., 45., 90., and 135.). Each GLCM feature descriptor was formed with 12 parameters computed based on the normalized matrix: mean, variance, entropy, angular second moment, energy, correlation, maximum probability, contrast, homogeneity, dissimilarity, cluster shade, and cluster prominence. This provided a GLCM feature descriptor of length 48. A spectral descriptor based on the Fourier spectra was computed specifically: firstkind statistical moments, spectral centroid within range and azimuth, spectral flux within range and azimuth, spectral rolloff, and the mean and variance of the first five cepstral coefficients [3]. This provided a spectral feature descriptor of length 17.

The same feature descriptors were used for different types of wavelet transforms. A descriptor $d$, which consisted of features $1-5,9$ was given by

$$
d_{j}=\left[\delta_{j, i}^{2}, \mu_{j, i},\left\|S_{j, i}\right\|_{2},\left\|S_{j, i}\right\|_{1}, E_{j, i}, D_{j}\right]
$$

where $j$ represented the decomposition level and $i$ belonged to the horizontal, vertical or diagonal details of wavelet decomposition. The coding gain was estimated for a whole image and was added as a single parameter at the end of a feature vector. 3 levels of dyadic decomposition were used $(j=3)$, therefore, $d$ had $3 \cdot((5 \cdot 3)+1)+1=49$ features for a DWT scenario, $3 \cdot((5 \cdot 6)+2)+1=97$ features for RODTWT scenario and $3 \cdot((5 \cdot 12)+4)+1=193$ ODTCWT transforms, respectively.

Tables I and II show the efficiency of the proposed methods using nonparametric and parametric approaches. The efficiency of the class recognition is measured as a percentage of the recognized images within a specific class using $10 \%$ and $20 \%$ images for the learning stages. Table I reports the results for nonparametric features obtained with different wavelet transforms: dyadic discrete wavelet transform (DWT), real dual tree oriented wavelet transform (RODTWT) and oriented dual
TABLE I

ACCURaCy of Class ReCOgNition in Percentage for NONPARAMETRIC FEATURES USING 10\% AND 20\% OF IMAGES FOR LEARNING STAGE

\begin{tabular}{|c||c|c|c|c|c|c|c|c|}
\hline \multicolumn{1}{|c||}{} & \multicolumn{7}{c|}{ Non-parametric features } \\
\cline { 2 - 9 } \multicolumn{1}{c|}{ Class } & \multicolumn{2}{|c|}{ DWT } & \multicolumn{2}{c|}{ RODTWT } & \multicolumn{2}{c|}{ ODTCWT } & GLCM & LC [5] \\
\cline { 2 - 9 } & $10 \%$ & $20 \%$ & $10 \%$ & $20 \%$ & $10 \%$ & $20 \%$ & $20 \%$ & $20 \%$ \\
\hline C1 & 80.6 & 82.3 & 83.5 & 87.1 & 87.4 & 97.1 & 39.8 & 92.5 \\
C2 & 66.6 & 72.3 & 70.1 & 75.4 & 90.7 & 96.2 & 60.6 & 90.7 \\
C3 & 90.1 & 71.5 & 92.1 & 93.1 & 95.1 & 97.3 & 34.1 & 81.9 \\
C4 & 88.1 & 90.3 & 90.2 & 92.7 & 97.8 & 100 & 49.2 & 95.4 \\
C5 & 70.3 & 86.4 & 90.6 & 90.6 & 85.6 & 93.9 & 58.9 & 95.4 \\
C6 & 84.4 & 94.3 & 72.5 & 93.7 & 98.2 & 100 & 44.5 & 95.7 \\
C7 & 93.6 & 96.3 & 86.1 & 99.9 & 100 & 100 & 37.3 & 80.8 \\
C8 & 90.6 & 100 & 95.3 & 99.7 & 98.5 & 100 & 53.8 & 89.7 \\
C9 & 80.4 & 98.6 & 90.8 & 100 & 92.8 & 96.3 & 50.8 & 87.5 \\
C10 & 83.2 & 100 & 80.2 & 100 & 97.1 & 100 & 65.4 & 87.0 \\
C11 & 86.2 & 88.1 & 99.3 & 93.8 & 86.9 & 100 & 71.1 & 94.5 \\
C12 & 77.9 & 96.3 & 88.1 & 96.0 & 83.3 & 92.7 & 70.7 & 78.0 \\
C13 & 49.8 & 67.6 & 79.9 & 71.8 & 87.5 & 90.8 & 36.6 & 79.8 \\
C14 & 80.6 & 96.2 & 82.2 & 97.3 & 91.3 & 96.5 & 30.4 & 67.5 \\
C15 & 86.1 & 99.8 & 89.2 & 100 & 95.5 & 97.8 & 43.4 & 63.2 \\
C16 & 89.6 & 93.2 & 90.8 & 92.2 & 99.0 & 100 & 40.9 & 93.2 \\
C17 & 90.3 & 94.3 & 80.7 & 97.6 & 100 & 100 & 47.3 & 76.4 \\
C18 & 96.2 & 100 & 92.2 & 100 & 100 & 100 & 50.83 & 89.0 \\
C19 & 93.6 & 96.2 & 96.7 & 95.4 & 100 & 100 & 89.3 & 94.3 \\
C20 & 96.7 & 98.5 & 97.2 & 100 & 92.6 & 100 & 47.96 & 96.9 \\
\hline total & 84.5 & 91.1 & 86.01 & 93.6 & 92.6 & 97.94 & 51.4 & 86.2 \\
\hline
\end{tabular}

tree complex wavelet transform (ODTCWT). Features obtained within different types of wavelet transform were computed in the same manner, but they varied in the total number of features. The average recognition rates for the wavelet transform based methods were generally better compared to the GLCM method [10] and log comulants [4] of the Fractional Fourier transform methods. The DWT-based patch categorization achieved $84 \%$ of recognition rate for the used database, when $10 \%$ of data were used at the learning stage and $91.1 \%$ when $20 \%$ of data were used at the learning stage. All classes had very similar recognition rates (approximately between 80 and $90 \%$ ). Classes $\mathrm{C} 2$ and $\mathrm{C} 13$ had lower recognition rates. The RODTWT improved the recognition rate and achieved 86 and 96\% of recognition when 10 and $20 \%$ of images were used for the learning stages, respectively. The ODTCWT was more sensitive to the orientations, had more subbands, which could better decorrelate energy, and it achieved 92 and $97 \%$ of recognition rate when 10 and $20 \%$ of the images were used during the learning stages, respectively. Table II shows the recognition rates of the parametric features within different wavelet transform domains using GMRF, computed using a linear model. Over-all the recognition rate was smaller when the parametric features were used. The best recognition rate was $87.8 \%$ with ODTCWT 
TABLE II

ACCuRACy of Class Recognition in Percentage for PARAMETRIC GMRF FEATURES USING 10\% AND 20\% OF IMAGES FOR LEARNING STAGE

\begin{tabular}{|c||c|c|c|c|c|c|}
\hline \multicolumn{1}{|c||}{} & \multicolumn{5}{c|}{ Parametric features GMRF } \\
\cline { 2 - 7 } Class & \multicolumn{2}{|c}{ DWT } & \multicolumn{2}{c|}{ RODTWT } & \multicolumn{2}{c|}{ ODTCWT } \\
\cline { 2 - 7 } & $10 \%$ & $20 \%$ & $10 \%$ & $20 \%$ & $10 \%$ & $20 \%$ \\
\hline \multirow{2}{*}{ C1 } & 35.1 & 39.8 & 76.3 & 84.6 & 87.0 & 86.3 \\
C2 & 55.3 & 61.3 & 80.3 & 85.9 & 89.1 & 98.1 \\
C3 & 24.5 & 34.6 & 70.9 & 77.1 & 80.3 & 87.2 \\
C4 & 37.6 & 45.5 & 69.5 & 75.5 & 72.7 & 84.4 \\
C5 & 55.1 & 63.4 & 65.5 & 74.6 & 69.1 & 82.5 \\
C6 & 40.5 & 48.0 & 68.4 & 71.9 & 75.0 & 79.4 \\
C7 & 33.5 & 42.0 & 66.5 & 71.1 & 73.4 & 74.7 \\
C8 & 51.8 & 56.5 & 72.3 & 80.9 & 78.6 & 89.6 \\
C9 & 60.5 & 72.2 & 80.3 & 89.7 & 90.5 & 90.8 \\
C10 & 75.2 & 78.9 & 70.6 & 75.4 & 75.3 & 88.8 \\
C11 & 68.5 & 76.1 & 90.6 & 96.2 & 90.6 & 95.3 \\
C12 & 66.7 & 76.0 & 89.1 & 93.6 & 98.8 & 98.1 \\
C13 & 44.9 & 53.2 & 60.5 & 71.3 & 65.8 & 77.9 \\
C14 & 34.1 & 43.5 & 67.3 & 71.0 & 75.9 & 76.2 \\
C15 & 55.3 & 61.5 & 73.6 & 83.8 & 78.5 & 83.5 \\
C16 & 33.5 & 42.8 & 88.5 & 91.8 & 97.2 & 92.6 \\
C17 & 55.4 & 64.4 & 83.6 & 88.1 & 91 & 94.1 \\
C18 & 48.1 & 52.3 & 81.6 & 91.8 & 92.5 & 98.6 \\
C19 & 86.6 & 91.8 & 82.5 & 90.4 & 92.7 & 94.4 \\
C20 & 46.7 & 50.8 & 70.5 & 73.7 & 77.7 & 83 \\
\hline total & 50.58 & 57.8 & 75.4 & 81.9 & 82.3 & 87.8 \\
\hline \multicolumn{1}{|c||}{} & & & & & & \\
\hline
\end{tabular}

because this transform was the most sensitive to the orientations within the image and as well as the GMRF can exploit those properties. The GMRF model is usually used to characterize textures and for those small patches was not the more appropriate method. The GMRF parameters were estimated using a sliding window of $64 \times 64$ pixels within wavelet transform and the texture parameters were averaged for each subband. Each subband had a texture parameter of model order 4, which was represented with 10 parameters. A miss classification occurred within classes $\mathrm{C} 1-\mathrm{C} 4$ and class $\mathrm{C} 20$ was confused with classes $\mathrm{C} 13, \mathrm{C} 15$, and $\mathrm{C} 16-\mathrm{C} 19$, when $10 \%$ of data was used for both parametric and nonparametric approaches.

Self-similar features within SAR images represent scenes with forest, landscape, see clutters, some symmetric urban areas, etc. which are represented with categories $\mathrm{C} 02-$ Forest, C04-Vegetation and water-channel, C07-Urban-03, C08-Urban-04, and C19-Buoy in Sea, within the proposed database, shown in Fig. 2.

\section{CONCLUSION}

This letter presented a comparison between the parametric and nonparametric approaches for SAR image categorization using different types of 2-D discrete wavelet transforms: dyadic discrete wavelet transform, real oriented dual tree wavelet transform and oriented dual tree complex wavelet transform. The nonparametric features within the presented types of wavelet transforms better characterize the features of the image patches in comparison with the parametric features, which used a texture GMRF model. From among them the nonparametric features obtained within the oriented dual tree complex wavelet transform gave the best results.

\section{REFERENCES}

[1] C. Tison, J.-M. Nicolas, F. Tupin, and H. Maître, "New statistical model for Markovian classification of urban areas in high-resolution SAR images," IEEE Trans. Geosci. Remote Sens., vol. 42, no. 10, pp. 2046-2057, Oct. 2004.

[2] M. Hebar, D. Gleich, and Z. Cucej, "Autobinomial model for SAR image despeckling and information extraction," IEEE Trans. Geosci. Remote Sens., vol. 47, no. 8, pp. 2818-2835, Aug. 2009.

[3] A. Popescu, I. Gavat, and M. Datcu, "Contextual descriptors for scene classes in very high resolution SAR images," IEEE Geosci. Remote Sens. Lett., vol. 9, no. 1, pp. 80-84, Jan. 2012.

[4] J. Singh and M. Datcu, "SAR image categorization with log cumulants of the fractional Fourier transform coefficients," IEEE Trans. Geosci. Remote Sens., vol. 51, no. 12, pp. 5273-5282, Dec. 2013.

[5] C.-R. Shyu, M. Klaric, G. Scott, A. Barb, C. Davis, and K. Palaniappan, "GeoIRIS: Geospatial information retrieval and indexing system-Content mining, semantics modeling, and complex queries," IEEE Trans. Geosci. Remote Sens., vol. 45, no. 4, pp. 839-852, Apr. 2007.

[6] T. Li and M. Ogihara, "Toward intelligent music information retrieval," IEEE Trans. Multimedia, vol. 8, no. 3, pp. 564-574, Jun. 2006.

[7] X. Zhan, R. Zhang, D. Yin, and C. Huo, "SAR image compression using multiscale dictionary learning and sparse representation," IEEE Geosci. Remote Sens. Lett., vol. 10, no. 5, pp. 1090-1094, Sep. 2013.

[8] J. S. Vestergaard, A. L. Dahl, R. Larsen, and A. A. Nielsen, "Classification of polarimetric SAR data using dictionary learning," in Proc. Image Signal Process. Remote Sens. XVIII, 2012, vol. 8537, pp. 85370X$1-85370 \mathrm{X}-9$.

[9] C. He, L. Liu, L. Xu, M. Liu, and M. Liao, "Learning based compressed sensing for SAR image super-resolution," IEEE J. Sel. Topics Appl. Earth Observ. Remote Sens., vol. 5, no. 4, pp. 1272-1281, Aug. 2012.

[10] F. Albgretzen, Statistical Texture Measures Computed From Gray Level Co-Occurrence Matrices 2008. [Online]. Available: http://www.uio.no/ studier/emner/matnat/ifi/INF4300/h08/undervisningsmateriale/glcm.pdf

[11] M. Hearst, S. Dumais, E. Osman, J. Platt, and B. Scholkopf, "Support vector machines," IEEE Intell. Syst. Appl., vol. 13, no. 4, pp. 18-28, Jul./Aug. 1998.

[12] J.-M. Lina and L. Gagnon, "Image enhancement with symmetric daubechies wavelets," in Proc. SPIE, 1995, vol. 2569, pp. 169-207.

[13] I. Selesnick, R. Baraniuk, and N. Kingsbury, "The dual-tree complex wavelet transform," IEEE Signal Process. Mag., vol. 22, no. 6, pp. 123151, Nov. 2005.

[14] G. W. Wornell, Signal Processing With Fractals: A wavelet-Based Approach. Englewood Cliffs, NJ, USA: Prentice-Hall, 1996.

[15] I. Daubechies, Ten Lectures on Wavelets. Philadelphia, PA, USA: SIAM, 1992.

[16] R. Coifman and M. Wickerhauser, "Entropy-based algorithms for best basis selection," IEEE Trans. Inf. Theory, vol. 38, no. 2, pp. 713-718, Mar. 1992

[17] B. Banjanin, B. Gergič, P. Planinšič, and Z. Cucej, "Entropy-threshold method for best basis selection," Image Vis. Comput, vol. 19, no. 7, pp. 447-484, May 2001

[18] C. Parra, K. Iftekharuddin, and D. Rendon, "Wavelet based estimation of the fractal dimension in fbm images," in Conf. Proc. 1st Int. IEEE EMBS Conf. Neural Eng., 2003, pp. 533-536.

[19] I. Norros, "A storage model with self-similar input," Queueing Syst., vol. 16, no. 3/4, pp. 387-396, 1994.

[20] C. Bouman and K. Sauer, "A generalized Gaussian image model for edgepreserving map estimation," IEEE Trans. Image Process., vol. 2, no. 3, pp. 296-310, Jul. 1993.

[21] D. S. Sivia, Data Analysis: A Bayesian Tutorial. Oxford, U.K. Clarendon, 1996.

[22] R. Chellappa, S. Chatterjee, and R. Bagdazian, "Texture synthesis and compression using Gaussian-Markov random field models," IEEE Trans. Syst., Man, Cybern., vol. SMC-15, no. 2, pp. 298-303, Mar./Apr. 1985. 